\title{
Pressure-energy correlations and thermodynamic scaling in viscous Lennard-Jones liquids
}

\author{
D. Coslovich * \\ Institut für Theoretische Physik, Technische Universität Wien, \\ Wiedner Hauptstraße 8-10, A-1040 Wien, Austria and \\ Dipartimento di Fisica Teorica, Università di Trieste, Strada Costiera 11, 34100 Trieste, Italy \\ C. M. Roland \\ Naval Research Laboratory, Code 6120, Washington DC 20375-5342, USA
}

(Dated: June 5, 2018)

\begin{abstract}
We use molecular dynamics simulation results on viscous binary Lennard-Jones mixtures to examine the correlation between the potential energy and the virial. In accord with a recent proposal [U. R. Pedersen et al. Phys. Rev. Lett. 100, 015701 (2008)], the fluctuations in the two quantities are found to be strongly correlated, exhibiting a proportionality constant, $\Gamma$, numerically equal to one-third the slope of an inverse power law approximation to the intermolecular potential function. The correlation is stronger at higher densities, where interatomic separations are in the range where the inverse power law approximation is more accurate. These same liquids conform to thermodynamic scaling of their dynamics, with the scaling exponent equal to $\Gamma$. Thus, the properties of strong correlation between energy and pressure and thermodynamic scaling both reflect the ability of an inverse power law representation of the potential to capture interesting features of the dynamics of dense, highly viscous liquids.
\end{abstract}

PACS numbers: 61.43.Fs, 61.20.Lc, 64.70.Pf, 61.20.Ja

\section{INTRODUCTION}

The myriad phenomena accompanying the supercooling of liquids continue to intrigue condensed matter scientists. On approaching the glass transition, the equilibrium, metastable liquid is comprised of fast and slow moving molecules, which interchange their roles at times on the order of the structural relaxation. This makes the many-body dynamics inherently cooperative and thus too complex to be solved by a first-principles treatment. Various models have been developed to describe and interpret the dynamic properties, but none rise to the level of a predictive theory. Nevertheless, progress can be made by combining experiments that capture some defining characteristic of the dynamics with computer simulations able to provide a microscopic interpretation.

One effective simplification in describing the dynamics of glass-forming liquids is provided by the experimental observation that structural relaxation times $\tau_{\alpha}$ and transport coefficients, encompassing a range of thermodynamic conditions, superpose when plotted as a function of $\rho^{\gamma} / T$, where $T$ is temperature, $\rho$ density, and $\gamma$ a material constant [1, 2, 3]. This thermodynamic scaling of $\tau_{\alpha}$ has been demonstrated for dozens of molecular liquids and polymers [4] and extends with good accuracy from the high temperature Arrhenius region, through the

\footnotetext{
*Electronic address: coslovich@cmt.tuwien.ac.at

$\dagger$ Electronic address: roland@nrl.navy.mil

$\ddagger$ Copyright (2009) American Institute of Physics. This article may be downloaded for personal use only. Any other use requires prior permission of the author and the American Institute of Physics.
}

dynamic crossover, down to the glass transition 5]. The justification for the scaling law

$$
\tau_{\alpha} \sim \mathcal{F}\left(\rho^{\gamma} / T\right)
$$

draws from early work of Hoover and coworkers [6, 7], who investigated the properties of fluids having a pairwise additive intermolecular potential described by an inverse power law (IPL)

$$
u(r)=\epsilon(\sigma / r)^{m}
$$

in which $m$ is a constant, $r$ the intermolecular separation, and $\epsilon$ and $\sigma$ have respective dimensions of energy and length. Generally, the intermolecular potential for liquids is represented as a pair-wise additive interaction, with the steep repulsive part written as an exponential function or an IPL. An advantage of the IPL is that all reduced thermodynamic and dynamic properties of such a system can be expressed in terms of the variable $\rho^{m / 3} / T[\underline{6}, 6,8]$. Simulations of vitrifying liquids have often employed an IPL potential [9, 10, 11, 12]. Recent theoretical models of energy landscapes, relevant for description of the glass transition, are also based on Eq. (2) but with the addition of a density-dependent constant to account for the longrange attractive forces [13, 14, 15, 16].

At high densities the liquid structure is determined mainly by the repulsions 17], suggesting that if the repulsive potential were accurately described by an IPL, the local dynamics would depend only on $\rho^{m / 3} / T$, with the empirical scaling exponent $\gamma$ identified with $m / 3$. Support for this idea comes from recent simulations using an $m$-6 Lennard-Jones (LJ) intermolecular potential

$$
u(r)=4 \epsilon\left[\left(\frac{\sigma}{r}\right)^{m}-\left(\frac{\sigma}{r}\right)^{6}\right]
$$


Relaxation times and diffusion constants from these simulations conform to Eq. (1) but with a scaling exponent $\gamma$ that is not equal to $m / 3[18,19,20]$. For polymers this is due in some measure to the effect of the intramolecular part of the potential [21]. More generally, a systematic study of LJ particles [20] showed that $\gamma$ follows closely the steepness of the repulsive core. In the latter system, however, the scaling exponent $\gamma$ is always larger than $m / 3$, due to contributions of the attractive term in Eq. (3) [22, 23, 24]. $\gamma$ is equal to the slope of an IPL fitted over the typical distances of closest approach between particles probed in the highly viscous regime. These interatomic separations are defined by values of $r$ between the first nonzero value of the radial distribution function and the position of the half-height of the first peak [20].

In addition to giving rise to the scaling of the dynamics [Eq. (1)], adequacy of the IPL approximation for highly viscous liquids suggests the existence of a correlation between equilibrium fluctuations of the configurational parts of energy and pressure; i.e., between the potential energy $U$ and the virial $W$. This expectation follows from the exact correlation between fluctuations in $U$ and $W$ for an IPL [23]. Recently, Dyre and coworkers showed from simulations that the potential energy and the virial strongly correlate for various nonassociated materials, with correlation coefficients exceeding 0.9 23, 25]. Liquids exhibiting both thermodynamic scaling and correlation between $U$ and $W$ may exhibit other interesting properties, such as sufficiency of a single parameter to describe their temperature-dependent viscoelasticity [26, 27]. In this work we assess the correlation of $U$ and $W$ in viscous LJ liquids having different repulsive interactions; i.e., different $m$ in Eq. (3). These are the same LJ liquids previously shown to conform to thermodynamic scaling of their diffusion coefficients [20]. Thus, from our results we can test the conjecture of Pedersen et al. 25] that liquids whose dynamics follow the thermodynamic scaling are strongly correlating and vice versa.

\section{MODELS}

The models considered in this work consist of additive, equimolar binary mixtures of 500 particles interacting through the $m-6$ LJ potential

$$
u_{\alpha \beta}(r)=4 \epsilon_{\alpha \beta}\left[\left(\frac{\sigma_{\alpha \beta}}{r}\right)^{m}-\left(\frac{\sigma_{\alpha \beta}}{r}\right)^{6}\right]
$$

where $\alpha, \beta=1,2$ are indexes of species. The mixtures studied have a size ratio $\sigma_{11} / \sigma_{22}=0.64$, masses $m_{1} / m_{2}=1.0$, and a unique energy scale $\epsilon_{12} / \epsilon_{11}=$ $\epsilon_{22} / \epsilon_{11}=1$.0. We refer to them herein as AMLJ. As in previous work [20], we consider different repulsive interactions: $m=8,12,24$, and 36 . We also study two other LJ liquids having $m=12$ : one proposed by Wahnström [28], which we denote as WAHN, and the nonadditive mixture of Kob and Andersen [29]. This latter,

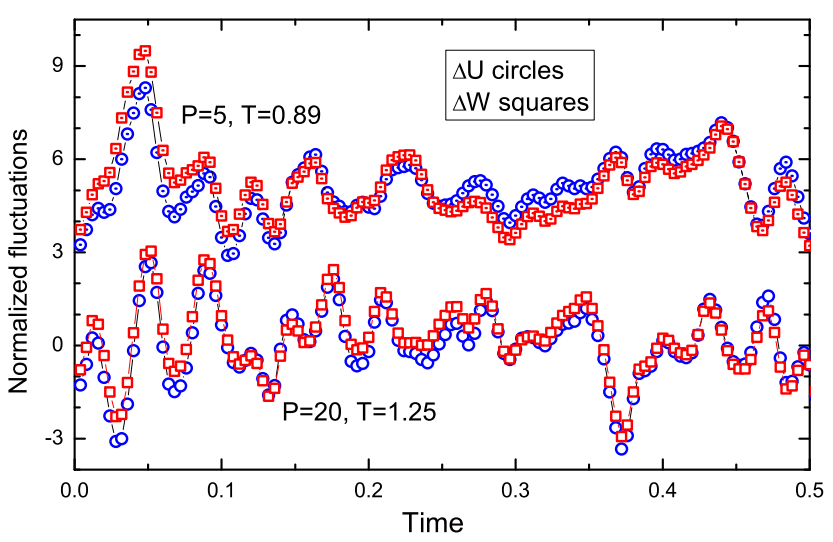

FIG. 1: Normalized instantaneous fluctuations of the potential energy $(U-\langle U\rangle) / \sqrt{\left\langle\Delta U^{2}\right\rangle}$ (circles) and virial $(W-$ $\langle W\rangle) / \sqrt{\left\langle\Delta W^{2}\right\rangle}$ (squares) for the AMLJ model with $m=36$ at the indicated pressure and temperature. The ordinate of the upper curves has been shifted 5 units for clarity.

called herein BMLJ, is among the most widely used in molecular dynamics simulations of the glass transition. In the following reduced LJ units will be used, assuming $\sigma_{11}, \epsilon_{11}$ and $\sqrt{m_{1} \sigma_{11}^{2} / \epsilon_{11}}$ as units of distance, energy and time respectively. All samples are cooled isobarically at pressures $P=5,10$, and 20 using Berendsen thermostat and barostat during equilibration. The production runs are then performed in the $N V E$ ensemble using the Velocity-Verlet algorithm. Further details on the simulations of AMLJ models can be found in Refs. [20, 30, 31].

We remark that the actual pressure varies with $m$ even though the numerical values of $P$ are the same, because the depth of the potential increases with $m$. A more appropriate set of reduction parameters for the potential in Eq. (4) is given by the position $r^{*}$ and the width $u^{*}=u_{11}\left(r^{*}\right)$ of the minima of $u_{11}(r)$. In terms of these parameters, the effective pressure is $P^{*}=P\left(u^{*} / \sigma^{* 3}\right)$, where $P$ is expressed in reduced LJ units. Considering the variation of $u^{*}$ and $\sigma^{*}$ for different $m$, the pressure $P$ for $m=36$, for example, should be increased by about a factor of 2.5 to match that of $m=12$.

\section{RESULTS}

Figure 1 is a plot of the instantaneous values of the normalized fluctuations in the virial and the potential energy for the AMLJ liquid with $m=36$ for two selected conditions of $T$ and $P$. As discussed below, this material exhibits the weakest correlation of $U$ and $W$ within the class of systems and range of state conditions investigated herein; nevertheless, a relationship between the two quantities is evident. The degree of correlation can be assessed by a variety of means. We calculate the 

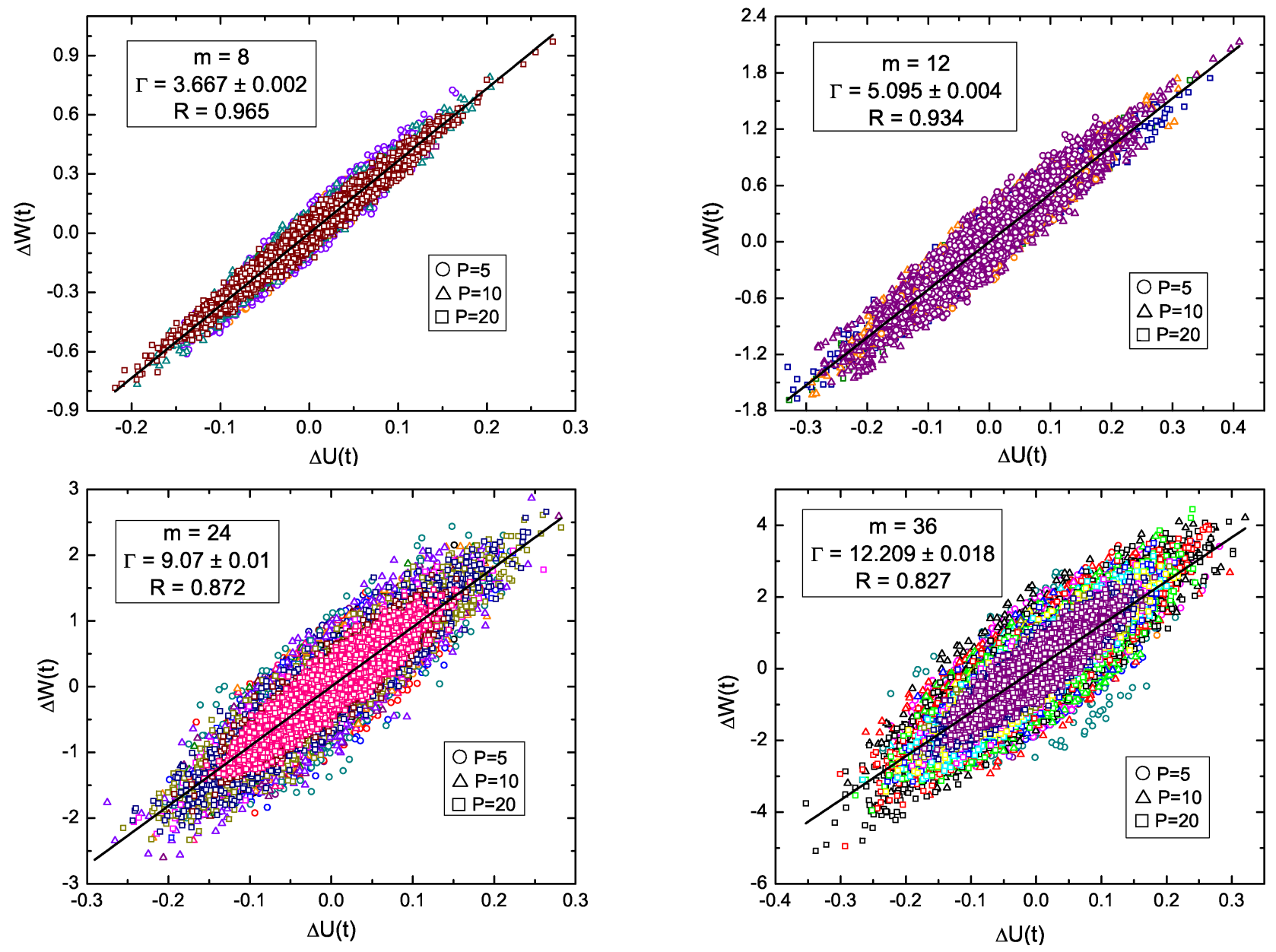

FIG. 2: Fluctuations of the virial versus those of the potential energy in the AMLJ model with $m=8,12,24$, and 36 . For each $m$, results for all pressures $P=5,10$, and 20 and all temperatures studied are shown. Colors in the online version indicate datasets corresponding to different state points. The corresponding range of densities is $1.04 \leq \rho \leq 2.06$ for $m=8$, $0.87 \leq \rho \leq 1.78$ for $m=12,0.93 \leq \rho \leq 1.70$ for $m=24$, and $0.77 \leq \rho \leq 1.71$ for $m=36$. The solid lines represents the least squares linear fits with the correlation coefficient $R$ indicated.

Pearson correlation coefficient

$$
R=\frac{\langle\Delta U \Delta W\rangle}{\sqrt{\left\langle(\Delta U)^{2}\right\rangle\left\langle(\Delta W)^{2}\right\rangle}}
$$

from linear regression of $\Delta U=U-\langle U\rangle$ and $\Delta W=$ $W-\langle W\rangle$, with the large number of data points, typically $2 \times 10^{5}$ per sample, enhancing statistical reliability. The determination of $R$ is carried out simultaneously for all state points over which thermodynamic scaling was observed in Ref. [20]; that is, for each pressure $(P=5$, 10 , and 20) at temperatures corresponding to normal liquid conditions down to the slow-dynamics regime.

In Fig. 2 the fluctuations in the virial are plotted versus those in the potential energy for AMLJ liquids with $m=8,12,24$, and 36 . The obtained $R$ are listed in Table I from which two observations can be made: The correlation coefficients are all close to unity, indicating substantial correlation. This concurs with the results of
Bailey et al. 23] for simulations of one-component LJ liquids with $m=12$ and of BMLJ particles. Second, the magnitude of $R$ decreases from $c a$. 0.97 to 0.83 with increasing magnitude of the repulsive exponent. This ostensibly suggests that liquids with steeper intermolecular repulsions are less strongly correlating. However, as discussed in Section III, the actual pressure regime explored by the particles in the simulation changes with $m$. Thus, larger $m$ corresponds to smaller effective $P$ and $\rho$. Since the correlation improves at higher density [23], reflecting the greater accuracy of the IPL approximation, the smaller $R$ for larger $m$ can be ascribed to the differences in thermodynamic conditions. We demonstrate this in Fig. 3, showing results for $m=36$ calculated for $P=30$ and 50. At these higher pressures the correlation coefficient is similar to the value of $R$ obtained for $m=12$ at $P=10$ and 20 . This confirms that the poorer correlation (smaller $R$ ) at higher $m$ is due to the lower 


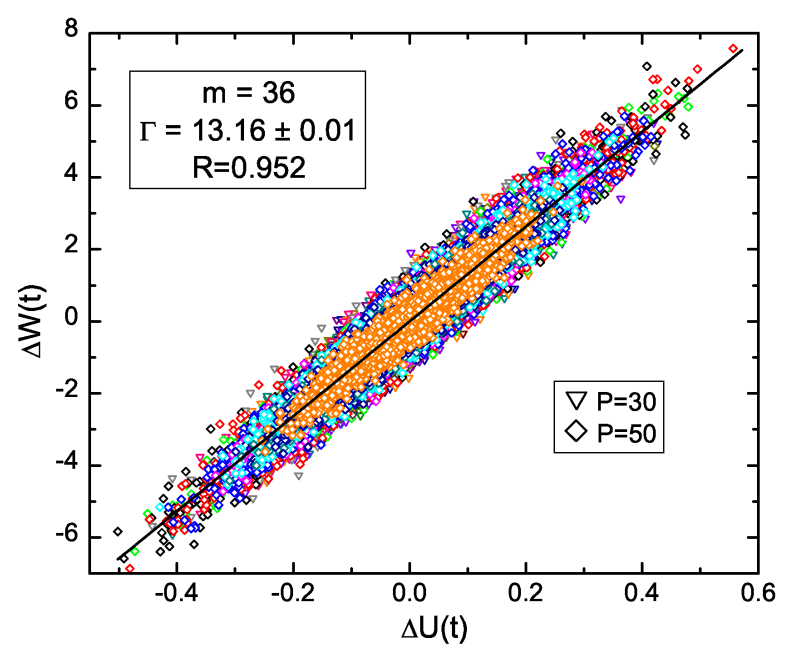

FIG. 3: Fluctuations of the virial versus those of the potential energy for the AMLJ model with $m=36$ at pressures $P=30$ and 50 and all $T$ studied (yielding $1.20 \leq \rho \leq 1.75$ ). These correspond to pressures $P^{*}$ (see Section [II) comparable to the values for $m=12$ at $P=10$ and 20 .

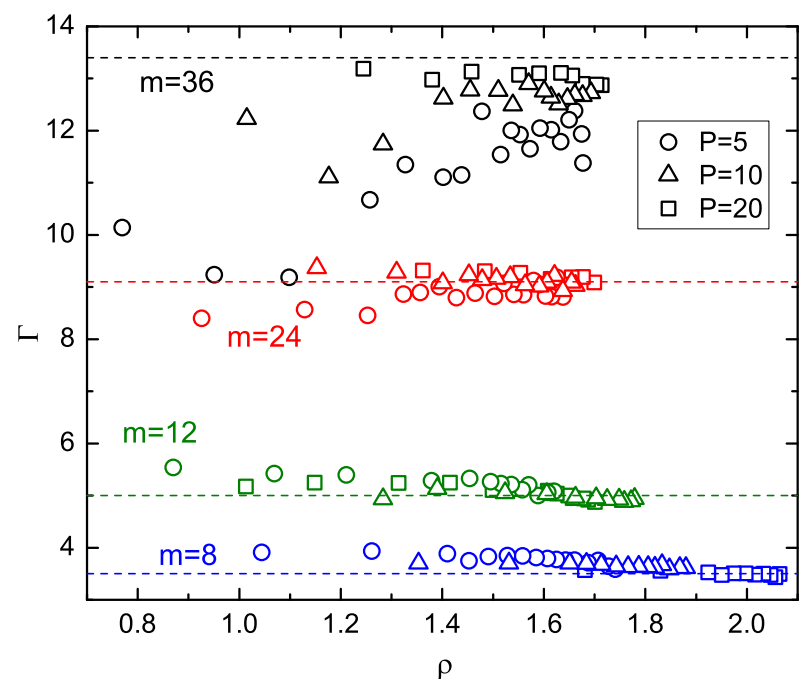

FIG. 4: Least squares slope $\Gamma$ calculated along individual isobars shown as a function of density $\rho=\rho(T, P)$. Results are shown for the AMLJ model with $m=8(0.18 \leq T \leq 2.0)$, $12(0.41 \leq T \leq 3.0), 24(0.75 \leq T \leq 4.0)$, and $36(0.87 \leq T \leq$ 6.0). The horizontal dashed lines denote the corresponding values of the scaling exponent $\gamma$ (from Ref. [20]).

effective pressure, and thus larger mean nearest neighbor distances, rather than to the steepness of repulsion per se.

For an exact IPL $\frac{d W}{d U}=m / 3$ [23] and regression of $W(U)$ yields the value of $m / 3$ as the slope of the fitted line. More generally, while the fact that $R \sim 1$ affirms correlation between $U$ and $W$, this is not a proof of proportionality of the two quantities [32]. However, it can be
TABLE I: Summary of exponents from $U-W$ correlations $(\Gamma)$ and from thermodynamic scaling of diffusion $(\gamma)$ for various LJ liquids. The correlation coefficient $R$ obtained from linear regression of $W(U)$ data is also included. Statistical uncertainties on $\Gamma$ correspond to one standard deviation in the "mean" case, and to the error associated to linear regression in the "global" case.

\begin{tabular}{lccccc}
\hline \hline & $m / 3$ & $\gamma$ & $\Gamma$ (global) & $R$ & $\Gamma$ (mean) \\
\hline AMLJ36 & 12 & $13.4 \pm 0.2$ & $12.21 \pm 0.02$ & 0.827 & $12.1 \pm 0.9$ \\
AMLJ24 & 8 & $9.1 \pm 0.1$ & $9.07 \pm 0.01$ & 0.872 & $9.0 \pm 0.2$ \\
AMLJ12 & 4 & $5.0 \pm 0.1$ & $5.095 \pm 0.004$ & 0.934 & $5.10 \pm 0.17$ \\
AMLJ8 & 2.7 & $3.5 \pm 0.1$ & $3.667 \pm 0.002$ & 0.965 & $3.67 \pm 0.13$ \\
BMLJ & 4 & $5.0 \pm 0.1$ & $5.087 \pm 0.003$ & 0.943 & $5.10 \pm 0.15$ \\
WAHN & 4 & $5.0 \pm 0.1$ & $5.052 \pm 0.003$ & 0.978 & $5.16 \pm 0.19$ \\
\hline \hline
\end{tabular}

observed that the scatter in the plots of Fig. 22 is normally distributed (random scatter without systematic trends), thereby justifying an interpretation of the slope, $\Gamma$, of the fitted lines as a measure of $m / 3$. Results for all simulations, including the BMLJ and WAHN, are listed in Table I, where it can be seen that there is good correspondence between the slope $\Gamma$ and the scaling exponent $\gamma$; that is, strongly correlating liquids conform to thermodynamic scaling, thus confirming the results of Ref. [33].

We can examine the relationship between pressure and energy fluctuations in more detail by evaluating the correlation for each state point individually. Figure 4 shows $\Gamma(T, P)$, obtained from linear regression, as a function of $\rho=\rho(T, P)$. Results for AMLJ systems with $m=8,12$, 24 , and 36 are shown in the figure, along with the scaling exponents (indicated by the dashed line) obtained from superpositioning of the diffusion constants for these liquids. Interestingly, at fixed $m, \Gamma(T, P)$ essentially collapse onto a single horizontal curve when plotted versus $\rho$, with only some variation at lower $T$ and $P$. These changes in $\Gamma(T, P)$ reflect the fact that the IPL approximation depends weakly on the state point, with the fluctuations of $\Gamma(T, P)$ for different state points mostly dictated by density variations (again excepting $m=36$ for the reasons discussed above). For each $m$, the mean of $\Gamma(T, P)$ over all state conditions is equivalent within the error to the $\Gamma$ obtained from a global fit of $\Delta W$ versus $\Delta U$ (see Table \).

One final comment concerns the link between the $\Gamma$ from the $U-W$ correlations and the thermodynamic scaling exponent $\gamma$. In Ref. [20] we found that a single value of $\gamma$ gave excellent superpositioning of the dynamic data. This means that any change in "local" $\gamma$ with $T$ and $P$ must be small (this issue was examined quantitatively for simulated liquid silica data in Ref. 34]). Even for the more poorly correlating liquid $(m=36)$, the estimated uncertainty in $\gamma$ is only about $15 \%( \pm 0.2)$. On the other hand, the slopes describing $U-W$ correlations display a somewhat wider variation upon changing state parameters, especially at low density and temperature. This 
may be related to the sensitivity of pressure-energy fluctuations to the shape of the pair potential for distances $r$ around and beyond the first peak in the radial distribution function (e.g., departures from the IPL form) [24]. Further investigations on the role of the attractive tail of the potential on $U-W$ correlations may be required to clarify this point.

\section{CONCLUSIONS}

In summary, we have shown that for viscous $m-6$ LJ liquids conforming to thermodynamic scaling of their dynamics, there is a strong correlation between the virial and the potential energy. This property is maintained for systems ranging from relatively soft particles $(m=8)$ to those approaching the hard sphere limit $(m=36)$. The correlation deteriorates for lower densities, as the range of the fluctuations extends to large $r$, for which the IPL approximation breaks down. The slopes ob- tained from linear regression of the virial vs. potential energy data are in good agreement with the scaling exponents yielding superpositioning of dynamic data, supporting the conjecture that pressure-energy correlations and thermodynamic scaling have a common origin in the IPL approximation of the interaction potential. Our results are in accord with the recent work of Dyre and coworkers 23, 24, 25, 33] on LJ particles and suggest the utility of the IPL approximation in describing essential features of the dynamics of dense, highly viscous liquids.

\section{Acknowledgments}

Computational resources were obtained through a grant within the agreement between the University of Trieste and CINECA (Italy). D. C. acknowledges financial support by the Austrian Science Fund (FWF) (Project number: P19890-N16). The work at NRL was supported by the Office of Naval Research.
[1] R. Casalini and C. M. Roland, Phys. Rev. E 69, 062501 (2004).

[2] C. Alba-Simionesco, A. Cailliaux, A. Alegria, and G. Tarjus, Europhys. Lett. 68, 58 (2004).

[3] C. Dreyfus, A. Le Grand, J. Gapinski, W. Steffen, and A. Patkowski, Eur. J. Phys. 42, 309 (2004).

[4] C. M. Roland, S. Hensel-Bielowka, M. Paluch, and R. Casalini, Rep. Prog. Phys. 68, 1405 (2005).

[5] R. Casalini and C. M. Roland, Phys. Rev. B 71, 014210 (2005).

[6] W. G. Hoover and M. Ross, Contemp. Phys. 12, 339 (1971).

[7] W. G. Hoover, S. G. Gray, and K. W. Johnson, J. Chem. Phys. 55, 1128 (1971).

[8] Y. Hiwatari, H. Matsuda, T. Ogawa, N. Ogita, and A. Ueda, Progr. Theor. Phys. 52, 1105 (1974).

[9] B. Bernu, J. P. Hansen, Y. Hiwatari, and G. Pastore, Phys. Rev. A 36, 4891 (1987).

[10] T. Grigera, A. Cavagna, I. Giardina, and G. Parisi, Phys. Rev. Lett. 88, 055502 (2002).

[11] C. De Michele, F. Sciortino, and A. Coniglio, J. Phys.: Condens. Matter 16, L489 (2004).

[12] L. A. Fernandez, V. Martin-Mayor, and P. Verrocchio, Phys. Rev. Lett. 98, 085702 (2007).

[13] P. G. Debenedetti, F. H. Stillinger, T. M. Truskett, and C. J. Roberts, J. Phys. Chem. B 103, 7390 (1999).

[14] M. S. Shell, P. G. Debenedetti, E. La Nave, and F. Sciortino, J. Chem. Phys. 118, 8821 (2003).

[15] R. J. Speedy, J. Phys.: Condens. Matter 15, S1243 (2003).

[16] M. S. Shell and P. G. Debenedetti, Phys. Rev. E 69, 051102 (2004).

[17] J. D. Weeks, D. Chandler, and H. C. Andersen, J. Chem. Phys. 54, 5237 (1971).

[18] J. Budzien, J. D. McCoy, and D. B. Adolf, J. Chem.
Phys. 121, 10291 (2004).

[19] G. Tsolou, V. A. Harmandaris, and V. G. Mavrantzas, J. Chem. Phys. 124, 084906 (2006).

[20] D. Coslovich and C. M. Roland, J. Phys. Chem. B 112, 1329 (2008).

[21] C. M. Roland, S. Bair, and R. Casalini, J. Chem. Phys. 125, 124508 (2006).

[22] D. Ben-Amotz and G. Stell, J. Chem. Phys. 119, 10777 (2003).

[23] N. P. Bailey, U. R. Pedersen, N. Gnan, T. B. Schrøder, and J. C. Dyre, J. Chem. Phys. 129, 184507 (2008).

[24] N. P. Bailey, U. R. Pedersen, N. Gnan, T. B. Schrøder, and J. C. Dyre, J. Chem. Phys. 129, 184508 (2008).

[25] U. R. Pedersen, N. P. Bailey, T. B. Schrøder, and J. C. Dyre, Phys. Rev. Lett. 100, 015701 (2008).

[26] N. L. Ellegaard, T. Christensen, P. V. Christiansen, N. B. Olsen, U. R. Pedersen, T. B. Schrøder, and J. C. Dyre, J. Chem. Phys. 126, 074502 (2007).

[27] N. P. Bailey, T. Christensen, B. Jakobsen, K. Niss, N. B. Olsen, U. R. Pedersen, T. B. Schrøder, and J. C. Dyre, J. Phys.: Condens. Matter 20, 244113 (2008).

[28] G. Wahnström, Phys. Rev. A 44, 3752 (1991).

[29] W. Kob and H. C. Andersen, Phys. Rev. E 51, 4626 (1995).

[30] D. Coslovich and G. Pastore, J. Chem. Phys. 127, 124504 (2007).

[31] D. Coslovich and G. Pastore, J. Chem. Phys. 127, 124505 (2007).

[32] F. J. Anscombe, Am. Stat. 27, 17 (1973).

[33] T. B. Schrøder, U. R. Pedersen, and J. C. Dyre, arXiv:0803.2199 (2008).

[34] A. Le Grand, C. Dreyfus, C. Bousquet, and R. M. Pick, Phys. Rev. E 75, 061203 (2007). 